\title{
Iptakalim Preferentially Decreases Nicotine-induced Hyperlocomotion in Phen- cyclidine-sensitized Rats: A Potential Dual Action against Nicotine Addiction and Psychosis
}

\author{
Nick Volf ${ }^{1}$, Gang $\mathrm{Hu}^{2}$, Ming $\mathrm{Li}^{1}$ \\ 'Department of Psychology, University of Nebraska-Lincoln, Lincoln, NE, USA, 'Jiangsu Key Laboratory of Neurodegeneration, Department \\ of Pharmacology, Nanjing Medical University, Nanjing, China
}

\begin{abstract}
Objective: Iptakalim is a putative ATP-sensitive potassium ( $\mathrm{K}_{\text {ATP }}$ ) channel opener. It is also a novel nicotinic acetylcholine receptor (nAChR) blocker and can antagonize nicotine-induced increase in dopamine release in the nucleus accumbens. Our recent work also shows that iptakalim exhibits a clozapine-like atypical antipsychotic profile, indicating that iptakalim may possess a dual action against nicotine addiction and schizophrenia.

Methods: The present study examined the potential therapeutic effects of iptakalim on nicotine use in schizophrenia. We created an animal model of comorbidity of nicotine addiction and schizophrenia by injecting male Sprague-Dawley rats with nicotine $(0.40 \mathrm{mg} / \mathrm{kg}$, subcutaneously [sc] ) or saline, in combination with phencyclidine (PCP, $3.0 \mathrm{mg} / \mathrm{kg}, \mathrm{sc}$ ) or saline daily for $14 \mathrm{con}-$ secutive days.

Results: During the PCP/nicotine sensitization phase, PCP and nicotine independently increased motor activity over time. PCP also disrupted prepulse inhibition (PPI) of acoustic startle response. Acute nicotine treatment attenuated the PCP-induced hyperlocomotion and PCP-induced disruption of PPI, whereas repeated nicotine treatment potentiated these effects. Importantly, pretreatment with iptakalim $(10-20 \mathrm{mg} / \mathrm{kg}$, intraperitoneally) reduced nicotine-induced hyperlocomotion in a dose-dependent fashion. This reduction effect was highly selective: it was more effective in rats previously sensitized to the combination of PCP and nicotine, but less effective in rats sensitized to saline, nicotine alone or PCP alone.

Conclusion: To the extent that the combined nicotine and PCP sensitization mimics comorbid nicotine addiction in schizophrenia, the preferential inhibitory effect of iptakalim on nicotine-induced hyperlocomotion suggests that iptakalim may be a potential useful drug for the treatment nicotine abuse in schizophrenia.
\end{abstract}

KEY WORDS: Iptakalim; Phencyclidine; Nicotine; Motor activity; Prepulse inhibition; Rats.

\section{INTRODUCTION}

Clinical observations suggest that there is a serious comorbidity of nicotine use and schizophrenia. ${ }^{1)}$ Schizophrenic patients also tend to be heavy smokers, defined as those who smoke more than one and a half packs a day. ${ }^{2)}$ They are also less likely to attempt quitting ${ }^{3)}$ and have a higher risk of developing smoking-related illnesses, such as lung cancer and cardiovascular disease. ${ }^{4)}$ Thus, a drug that could reduce nicotine use while maintaining antipsychotic efficacy in the treatment of schizophrenia

\footnotetext{
Received: April 16, 2012 / Revised: May 15, 2012

Accepted: May 17, 2012

Address for correspondence: Ming Li, PhD

Department of Psychology, University of Nebraska-Lincoln, 238

Burnett Hall, Lincoln, NE 68588-0308, USA

Tel: +1-402-472-3144, Fax: +1-402-472-4637

E-mail: mli2@unl.edu
}

would be highly desirable. Unfortunately, all currently used antipsychotics except clozapine, ${ }^{5)}$ are ineffective in this regard. Since clozapine use is limited because of its risk in causing life-threatening agranulocytosis, there is an urgent need to search and evaluate other effective pharmacological treatments with novel mechanisms of action.

Iptakalim is a novel adenosine triphosphate (ATP)-sensitive potassium $\left(\mathrm{K}_{\mathrm{ATP}}\right)$ channel activator that was originally developed for the treatment of hypertension. ${ }^{6}$ It can pass easily through the blood-brain-barrier and shows a potential neuroprotective effect for neurons and astrocytes against ischaemia, trauma and neurotoxins. ${ }^{7,8)}$ Iptakalim is also a novel nicotinic acetylcholine receptor (nAChR) blocker and is shown to antagonize nicotine-induced increase in dopamine release in the nucleus accumbens. ${ }^{8,9)}$ Our recent work shows that iptakalim also possesses a clozapine-like antipsychotic activity. ${ }^{10)}$ We found that iptakalim is effective in reducing amphetamine- and phencycli-

(c) This is an Open-Access article distributed under the terms of the Creative Commons Attribution Non-Commercial License (http://creativecommons.org/licenses/by-nc/3.0) which permits unrestricted non-commercial use, distribution, and reproduction in any medium, provided the original work is properly cited. 
dine-induced hyperlocomotion as well as selectively disrupting conditioned avoidance responding, three well-established behavioral indices of antipsychotic activity. ${ }^{11,12)}$ Similar to clozapine, iptakalim preferentially increases c-Fos expression in the medial prefrontal cortex, nucleus accumbens and lateral septum. ${ }^{10)}$

Based on these findings, we hypothesized that iptakalim may be a drug with a dual action against nicotine use/abuse and psychosis. The objective of the present study was to provide a needed behavioral assessment of the potential therapeutic effects of iptakalim on nicotine use in schizophrenia using a preclinical animal approach. To establish an animal model of comorbidity of nicotine abuse in schizophrenia, we sensitized rats to phencyclidine (PCP, $3.0 \mathrm{mg} / \mathrm{kg}$, subcutaneously [sc]), in combination with nicotine $(0.4 \mathrm{mg} / \mathrm{kg}, \mathrm{sc})$ for 14 consecutive days. The PCP sensitization was chosen as a model of schizophrenia because it mimics many aspects of the illness, such as hypoglutamatergic activity, ${ }^{13)}$ prepulse inhibition (PPI) deficit, ${ }^{14)}$ persistent deficits in cognition, ${ }^{15)}$ social interaction impairment, ${ }^{16)}$ and increased depressive-like behavior during the forced swim test. ${ }^{17)}$ The effects of repeated PCP and/or nicotine treatment, and their interactions were assessed in a series of locomotor activity and PPI tests. The effects of iptakalim on nicotine-induced hyperlocomotion was taken as a measure of efficacy of iptakalim to ameliorate the psychomotor stimulant and/or rewarding effects of nicotine. ${ }^{18,19)}$

\section{METHODS}

\section{Animals}

Male Sprague-Dawley rats (226-260 g upon arrival; Charles River, Portage, MI, USA) were housed two per cage, in $48.3 \times 26.7 \times 20.3 \mathrm{~cm}$ transparent polycarbonate cages under 12-hour light/dark conditions (light on between 6:00 am and 6:00 pm). Room temperature was maintained at $22 \pm 1{ }^{\circ} \mathrm{C}$ with a relative humidity of $45-60 \%$. Food and water were available ad libitum. Animals were allowed at least one week of habituation to the animal facility before they were used in experiments. All procedures were approved by the Institutional Animal Care and Use Committee at the University of Nebraska-Lincoln.

\section{Motor Activity Monitoring Apparatus}

Each of the sixteen activity boxes $(48.3 \times 26.7 \times 20.3 \mathrm{~cm}$ transparent polycarbonate cages) was equipped with a row of 6 photocell beams $(7.8 \mathrm{~cm}$ between two adjacent photobeams) placed $3.2 \mathrm{~cm}$ above the floor of the cage.
Motor activity was recorded as the number of the photocell beam breaks by a custom-built program run on a computer.

\section{PPI of acoustic startle response apparatus}

The PPI test was performed using six Startle Monitor Systems (Kinder Scientific, Julian, CA, USA) controlled by a computer. Each system was housed in a compact sound attenuation cabinet $(35.56 \mathrm{~cm}$ wide $\times 27.62 \mathrm{~cm}$ deep $\times 49.53 \mathrm{~cm}$ high). A speaker (diameter: $11 \mathrm{~cm}$ ) mounted on the cabinet's ceiling was used to generate acoustic stimuli (70 dB-120 dB). The startle response was measured by a piezoelectric sensing platform on the floor, which was calibrated daily. During testing, rats were placed in a rectangular box made of transparent Plexiglas ( $19 \mathrm{~cm}$ wide $\times 9.8 \mathrm{~cm}$ deep $\times 14.6 \mathrm{~cm}$ high) with an adjustable ceiling positioned atop the box, providing only limited restraint while prohibiting ambulation.

\section{Drugs}

Doses of nicotine hydrogen tartrate (Sigma, St. Louis, MO, USA) were expressed as free base dissolved in $0.9 \%$ saline. The nicotine solution was brought to a $\mathrm{pH}$ of $7.0 \pm 0.2$ with a dilute $\mathrm{NaOH}$ solution. We chose the 0.4 $\mathrm{mg} / \mathrm{kg}$ nicotine dose because it is a commonly used dose in a number of behavioral tasks, such as motor activity, ${ }^{20)}$ conditioned place preference, ${ }^{21,22)}$ and working memory. ${ }^{23)}$ The injection solutions of phencyclidine hydrochloride (a gift from NIDA Chemical Synthesis and Drug Supply Program) and iptakalim hydrochloride (IPT, 99.9\%, a gift provided by the Institute of Pharmacology and Toxicology, Academy of Military Medical Sciences of China to Dr. Hu) were obtained by mixing drugs with $0.9 \%$ saline. Nicotine and PCP were administered subcutaneously at a volume of $1.0 \mathrm{ml} / \mathrm{kg}$. Iptakalim was administered intraperitoneally [ip], also at a volume of $1.0 \mathrm{ml} / \mathrm{kg} .{ }^{10}$ )

\section{Experimental Procedure}

1. Habituation and baseline PPI test (Days 1-2)

Sixty-four male Sprague-Dawley rats (2 batches of 32 ) were used. On the first day, all rats were habituated to both the locomotor activity (LMA) chambers and PPI chambers for 30 minutes and 20 minutes respectively. On the second day, rats were habituated to the LMA chambers, followed by a baseline of PPI test. The PPI procedure was adapted from Culm and Hammer. ${ }^{24)}$ Each session lasted approximately 18 minutes and began with a 5 minutes period of $70 \mathrm{~dB}$ background noise (which continued throu- 
ghout the duration of the session) followed by four different trial types: PULSE ALONE trials and three types of PREPULSE+PULSE trials, which consisted of a 20 mimiseconds (ms) 73, 76, or $82 \mathrm{~dB}$ prepulse $(3,6$, and $12 \mathrm{~dB}$ above background) followed $100 \mathrm{~ms}$ later by a $120 \mathrm{~dB}$ pulse. Each session was divided into 4 blocks. Blocks 1 and 4 were identical, each consisting of 4 PULSE ALONE trials. Blocks 2 and 3 were also identical and each consisted of 8 PULSE ALONE trials and 5 of each PREPULSE+PULSE trial type. A total of 54 trials were presented during each test session. Trials within each block were presented in a pseudorandom order and were separated by a variable intertrial interval averaging $15 \mathrm{sec}-$ onds (ranging from 9-21 seconds). Startle magnitude was defined as the maximum force (measured in Newtons) applied by the rat to the startle apparatus recorded over a period of $100 \mathrm{~ms}$ beginning at the onset of the pulse stimulus. Startle responses from testing Blocks 2 and 3 were used to calculate percent PPI (\%PPI) for each acoustic prepulse trial type:

$$
\% \mathrm{PPI}=100-\left[\left(\begin{array}{c}
\text { Average startle response to } \\
\text { PREPULSE+PULSE trials } \\
\hline \begin{array}{c}
\text { Average statle response to } \\
\text { PULSE ALONE trials }
\end{array}
\end{array}\right) \times 100\right]
$$

\section{Sensitization induction by repeated PCP and/or nicotine treatment (Days 3-16)}

After two days of habituation, the rats were randomly assigned to one of four groups (saline + saline $[\mathrm{SAL}+\mathrm{SAL}]$, saline+nicotine $[\mathrm{SAL}+\mathrm{NIC}], \mathrm{PCP}+$ saline $[\mathrm{PCP}+\mathrm{SAL}]$, and $\mathrm{PCP}+$ nicotine $[\mathrm{PCP}+\mathrm{NIC}], \mathrm{n}=16$ /group). Every day for 14 days, the rats were brought into the lab in their home cages, allowed to habituate for 30 minutes, and then injected with either PCP $(3.0 \mathrm{mg} / \mathrm{kg}, \mathrm{sc})$ or saline. This dose of PCP was chosen because it has been shown to produce a robust behavioral sensitization and disruption of learning. ${ }^{25-27)}$ Five minutes later a second injection of either nicotine (0.4 $\mathrm{mg} / \mathrm{kg}$, sc) or saline was administered. This sensitization phase lasted for 14 consecutive days. On Days 1, 6, 11 and 13 , the rats were also placed in the LMA chambers $4 \mathrm{mi}-$ nutes after the second injection. LMA was recorded for 90 minutes. On Days 3, 8, 12 and 14, the rats were placed in the PPI chambers 5 minutes after the second injection and their PPI performance was tested. On days when rats were not tested, rats were injected in their home cages only.

\section{PCP challenge test (Day 23) and iptakalim test on nicotine-induced hyperlocomotion in the same LMA boxes (Day 25)}

Seven days after the last $\mathrm{PCP} /$ nicotine injections, all rats received a challenge dose of $\mathrm{PCP}(1.5 \mathrm{mg} / \mathrm{kg}, \mathrm{sc})$ and were tested in the LMA chambers to assess the PCP-induced psychomotor sensitization for 90 minutes. Two days later, the effects of iptakalim treatment on nicotine-induced hyperlocomotion were assessed. Each of the original four groups was further divided into three subgroups: saline ( $\mathrm{n}=8)$, iptakalim $(10 \mathrm{mg} / \mathrm{kg}, \mathrm{ip}, \mathrm{n}=4)$, or iptakalim $(20 \mathrm{mg} / \mathrm{kg}, \mathrm{ip}, \mathrm{n}=4)$. At the beginning of the test, rats were first injected with iptakalim or saline and $5 \mathrm{mi}-$ nutes later received a challenge dose of nicotine $(0.2$ $\mathrm{mg} / \mathrm{kg}, \mathrm{sc})$. Four minutes after receiving nicotine, rats were placed in the LMA chambers and motor activity was recorded for 90 minutes.

\section{Iptakalim test on nicotine-induced hyperlocomotion in a novel environment (Day 29)}

Four days after the first iptakalim test in the familiar LMA boxes, the effects of iptakalim treatment on nicotine-induced increase in motor activity were assessed in a novel environment to assess the generality of the iptakalim effect on nicotine-induced hyperlocomotion. Rats were tested in 8 two-compartment chambers $(64 \mathrm{~cm}$ wide $\times 30 \mathrm{~cm}$ high $\times 24 \mathrm{~cm}$ deep) housed in a ventilated, sound-insulated isolation cubicle $(96.52 \mathrm{~cm}$ wide $\times 35.56$ $\mathrm{cm}$ deep $\times 63.5 \mathrm{~cm}$ high; Med Associates, St. Albans, VT, USA). The motor activity was detected by a set of 16 photobeams (ENV-256-8P; Med Associates, St. Albans, VT, USA) affixed at the bottom of the box $(3.5 \mathrm{~cm}$ above the grid floor) as number of beam breaks. The groups were the same as in the first iptakalim test. At the beginning of the test, rats received the first injection of either saline, iptaka$\lim (10 \mathrm{mg} / \mathrm{kg}$, ip), or iptakalim (20 mg/kg, ip), followed by a challenge dose of nicotine $(0.2 \mathrm{mg} / \mathrm{kg}, \mathrm{sc}) 5$ minutes later. The motor activity test started immediately after the nicotine injection and lasted for 30 minutes.

\section{Data Analysis}

All data were expressed as mean+SEM. Motor activity from the PCP/nicotine sensitization induction phase were analyzed using repeated-measures analyses of variance (ANOVAs) with a within-subjects factor of test day and between-subjects factors of $P C P$ and nicotine treatment. Percent PPI data at the three prepulse intensity levels (e.g., 73,76 and $82 \mathrm{~dB}$ ) on the 4 drug testing days were presented separately and analyzed similarly with the ex- 
ception that the prepulse level was added as a within-subjects factor. One-way ANOVA followed by post hoc least significant difference (LSD) tests was used to determine group differences on specific test days. Motor activity data from the PCP challenge test were analyzed by a two-way ANOVA with $P C P$ and nicotine as two between-subjects factors. Iptakalim data from the two nicotine challenge days were analyzed separately by a three-way ANOVA with iptakalim, PCP and nicotine as three between-subjects factors.

\section{RESULTS}

\section{LMA during the Sensitization Induction Phase}

Data for one rat in the SAL+SAL group on the last motor activity test was missing due to a technical error and were not entered in the analysis. As can be seen in Fig. 1, both PCP and nicotine treatment progressively increased motor activity throughout the four test days. Repeated measures ANOVA revealed a main effect of $P C P, F(1$, $59)=332.68, p<0.001$, and nicotine, $F(1,59)=12.88, p=$ 0.001 , but no $P C P \times$ nicotine interaction, $F(1,59)=1.7879$, $p=0.187$. Both PCP and nicotine also induced behavioral sensitization as evidenced by the significant $P C P \times$ test day, $F(3,177)=11.104, p<0.001$, and nicotine $\times$ test day interaction, $F(3,177)=16.980, p<0.001$. One-way ANOVA followed by the post hoc LSD tests on each test day showed that both PCP groups showed greater numbers of activity

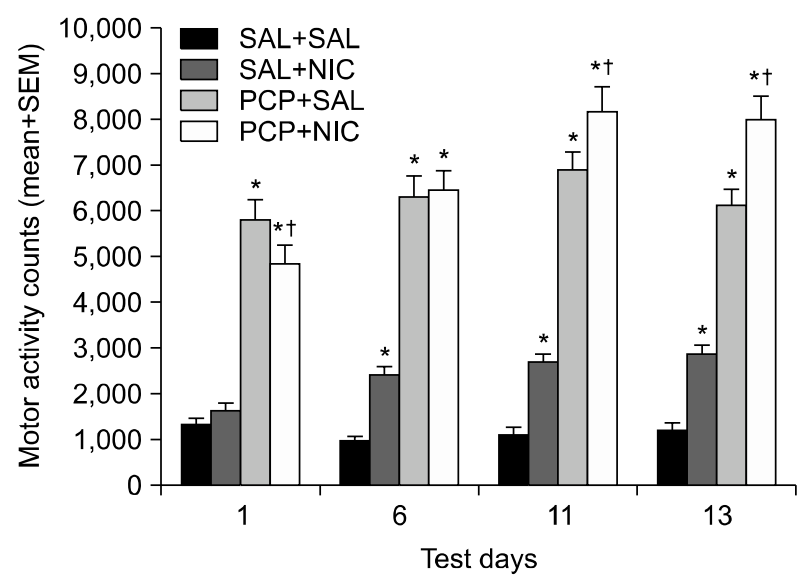

Fig. 1. Effects of repeated administration of SAL, PCP $(3.0 \mathrm{mg} / \mathrm{kg}$, $\mathrm{sc})$, and $\mathrm{NIC}(0.4 \mathrm{mg} / \mathrm{kg}, \mathrm{sC})$ on locomotor activity (mean+SEM). A total of 64 rats were randomly assigned to one of four groups $(n=16 /$ group): $S A L+S A L, S A L+N I C, P C P+S A L$, and PCP+NIC and tested on day $1,6,11$ and 13 of drug treatment. ${ }^{*} p<0.05$ significantly different from the SAL+SAL control group; ${ }^{\dagger} p<0.05$ significantly different between two PCP groups (NIC vs. SAL). SAL, saline; PCP phencyclidine hydrochloride; NIC, nicotine; SEM, standard error of the mean than the SAL+SAL group on all test days, $p<0.001$. Nicotine treatment alone also significantly increased motor activity, as evidenced by the significant group differences between the SAL+NIC and SAL+SAL groups on the last three days of testing, $p<0.011$. Interestingly, on the first day of testing, the PCP+NIC group had significantly lower motor activity than the $\mathrm{PCP}+\mathrm{SAL}$ group, $p=0.034$, suggesting an acute inhibitory effect of nicotine on PCP-induced hyperlocomotion. This effect was in contrast to the potentiated effect seen in the latter part of testing. The $\mathrm{PCP}+\mathrm{NIC}$ group showed significantly higher motor activity than the $\mathrm{PCP}+\mathrm{SAL}$ group on the last two testing days, $p=0.009$ and $p<0.001$, respectively.

\section{PPI during the Sensitization Induction Phase}

Data for two rats in the PCP+SAL group and two in the $\mathrm{SAL}+\mathrm{NIC}$ groups were missing due to a technical error and were not analyzed. Fig. 2 shows PPI performance of the four groups of rats tested throughout the sensitization phase. PCP $3.0 \mathrm{mg} / \mathrm{kg}$ disrupted PPI at all three levels. Analysis of PPI data from the 4 drug test days revealed a main effect of $P C P, F(1,56)=120.647, p<0.001$, but no main effect of nicotine, $F(1,56)=1.821, p=0.183$, nor $P C P \times$ nicotine interaction, $F(1,56)=0.086, p=0.770$. The effect of prepulse level was significant, $F(2,112)=512.147$, $p<0.001$, but the effect of test day was not, $F(3,168)=$ $2.085, p=0.104$. There were also significant interactions between $P C P \times$ test day, $F(3,168)=3.012, p=0.032, P C P \times$ prepulse level, $F(2,112)=17.611, p<0.001$, and nicotine $\times$ test day, $F(3,168)=3.768, p=0.012$, suggesting that the effects of PCP and nicotine on PPI performance varied across the test days.

At the $73 \mathrm{~dB}$ prepulse level, one-way ANOVA followed by post hoc LSD tests revealed that the two PCP groups had significantly lower PPIs than the two SAL groups on all test days, $p<0.031$ except on the second PPI drug test day, the two PCP groups did not differ from the SAL $+\mathrm{SAL}$ group, $p>0.084$. At the $76 \mathrm{~dB}$ prepulse level, once again, the two PCP groups had significantly lower PPIs than the other two groups on all test days, $p<0.012$. Interestingly, the PCP+NIC group had even lower PPI on the last test day than the PCP+SAL group, $p=0.041$, suggesting that repeated nicotine treatment worsened the PPI-disruptive effect of PCP. At the $82 \mathrm{~dB}$ prepulse level, the two PCP groups also exhibited significantly lower PPIs than the two SAL groups, $p<0.002$. In addition, on the first PPI drug test day, the PCP+NIC group had significantly higher PPI on than the PCP+SAL group, $p=0.039$. However, this effect was reversed on the second drug test day, 

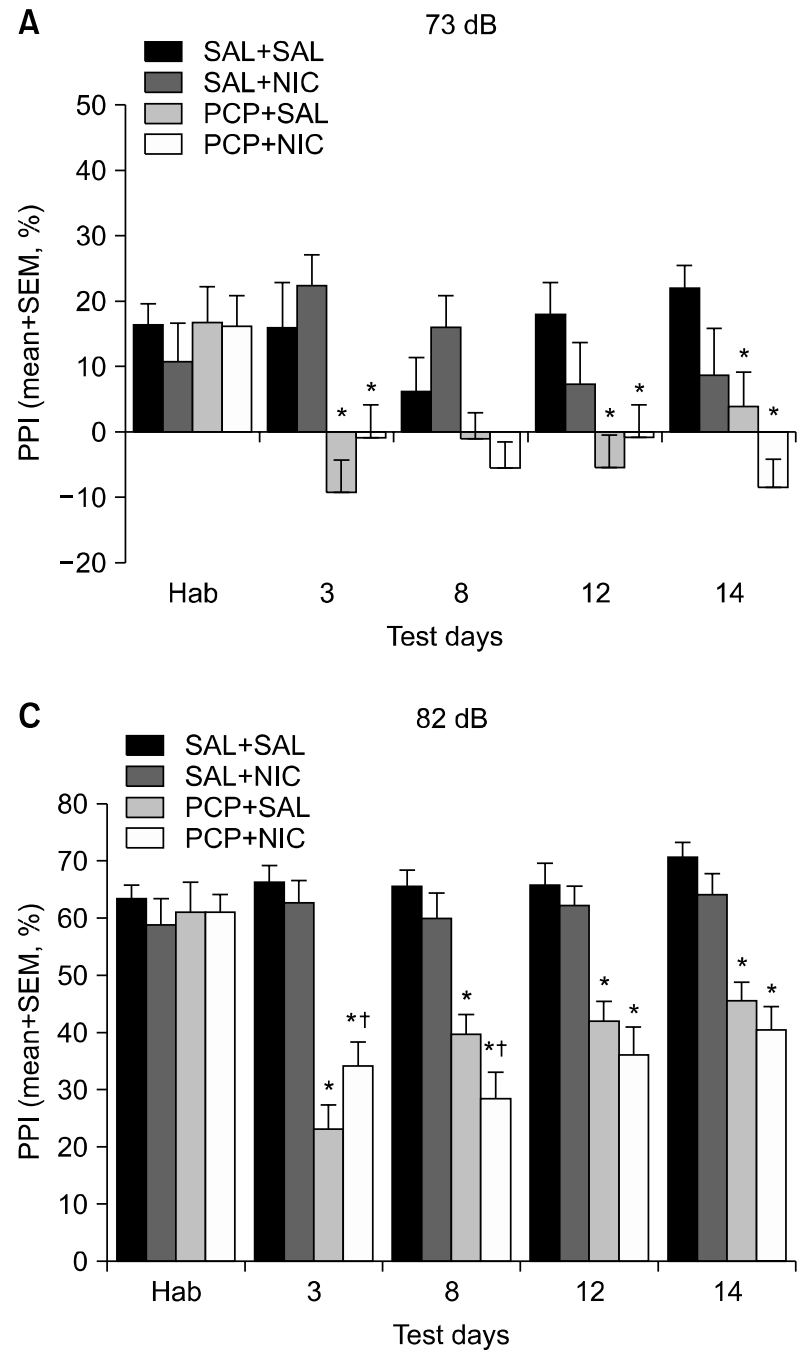

$p=0.037$. Inspection of the overall PPI patterns in the $\mathrm{PCP}+\mathrm{SAL}$ and $\mathrm{PCP}+\mathrm{NIC}$ groups across the four test days supports the observation that acute nicotine treatment tended to attenuate the PCP effect, while repeated treatment tended to worsen it. This pattern of nicotine effect on PCP in the PPI model is consistent with its effect on PCP-induced hyperlocomotion.

\section{PCP Sensitization Assessment}

Fig. 3 shows motor activity in the four groups of rats challenged with PCP $1.5 \mathrm{mg} / \mathrm{kg}$. Two-way ANOVA revealed a main effect of $P C P, F(1,60)=12.242, p=0.001$, confirming the PCP-induced behavioral sensitization. The nicotine effect was not significant, $F(1,60)=2.572$, $p=0.114$, indicating that repeated nicotine treatment did not have a long-term impact on PCP sensitization. One-way ANOVA followed by post hoc LSD tested on the four groups showed that both $\mathrm{PCP}+\mathrm{SAL}$ and $\mathrm{PCP}+\mathrm{NIC}$

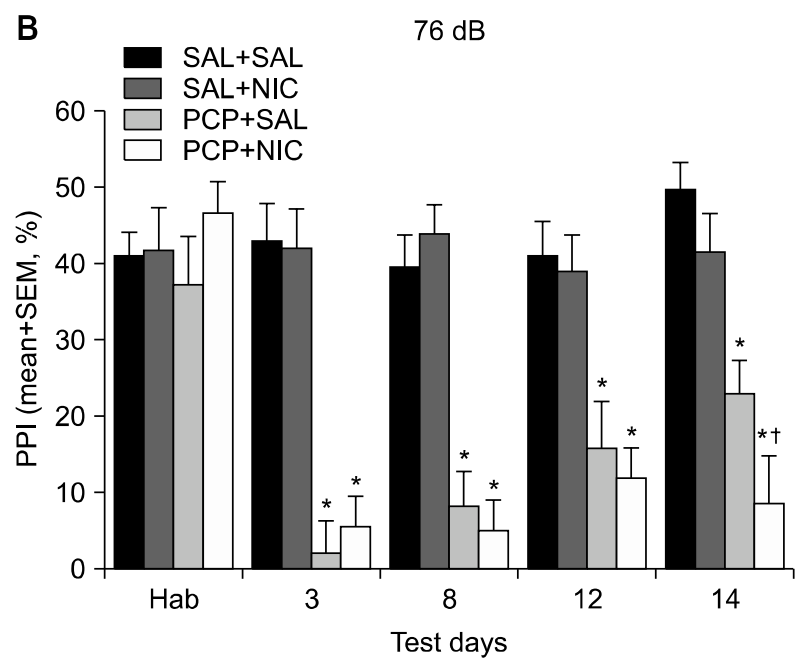

Fig. 2. Effects of repeated administration of $S A L, P C P(3.0 \mathrm{mg} / \mathrm{kg}$, $\mathrm{sc})$, and $\mathrm{NIC}(0.4 \mathrm{mg} / \mathrm{kg}, \mathrm{sc})$ on PPI of acoustic startle response at the $73 \mathrm{~dB}$ prepulse level (A), $76 \mathrm{~dB}$ prepulse level (B) and 82 $\mathrm{dB}$ prepulse level $(\mathrm{C})$ (mean+SEM). A total of 64 rats were randomly assigned to one of four groups ( $n=16 /$ group): $S A L+S A L$, $\mathrm{SAL}+\mathrm{NIC}, \mathrm{PCP}+\mathrm{SAL}$, and $\mathrm{PCP}+\mathrm{NIC}$ and tested on day $1,6,11$ and 13 of drug treatment. The PPI tests were conducted on the second habituation day (Hab), and on day $3,8,12$ and 14 of drug treatment. ${ }^{*} p<0.05$ significantly different from the $S A L+S A L$ control group; ${ }^{\dagger} p<0.05$ significantly different between two PCP groups (NIC vs. SAL). SAL, saline; PCP, phencyclidine; NIC, nicotine; PPI, prepulse inhibition; SEM, standard error of the mean.

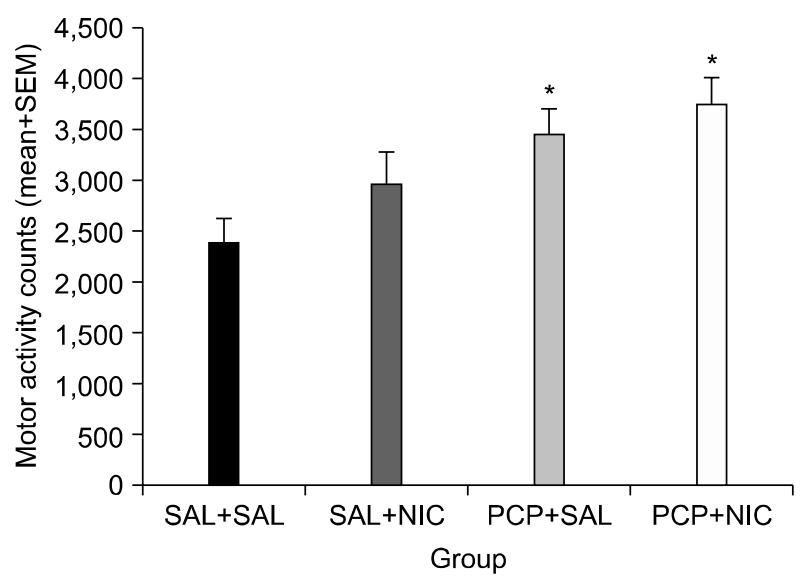

Fig. 3. Effects of repeated administration of SAL, PCP $(3.0 \mathrm{mg} / \mathrm{kg}$, $\mathrm{sc})$, and $\mathrm{NIC}(0.4 \mathrm{mg} / \mathrm{kg}, \mathrm{sC})$ on the expression of PCP locomotor sensitization during the PCP challenge test in the four groups of rats ( $n=16 /$ group) that were previously treated with $S A L+S A L$, $S A L+N I C, P C P+S A L$, and PCP+NIC. Each rat was injected with PCP $1.5 \mathrm{mg} / \mathrm{kg}, \mathrm{sc}$, and locomotor activity (mean+SEM) was recorded for 90 minutes. ${ }^{*} p<0.05$ significantly different from the $S A L+S A L$ control group. SAL, saline; PCP, phencyclidine; NIC, nicotine; SEM, standard error of the mean; SEM, standard error of the mean. 
groups showed significantly greater motor activity than the SAL+SAL group, $p<0.007$.

\section{Iptakalim test on nicotine-induced hyperlocomotion in the same LMA boxes}

To examine the time course of the iptakalim effect on nicotine-induced increase in motor activity, we analyzed motor activity in each of 30 minutes blocks (a total of 3 blocks) separately (Fig. 4). During the first 30 minutes of testing, three-way ANOVA revealed a main effect of nicotine, $F(1,52)=39.138, p<0.001$, suggesting that rats previously treated with nicotine expressed a nicotine behavioral sensitization. There was also a significant $P C P \times$ nicotine interaction, $F(1,52)=12.716, p=0.001$, indicating that the nicotine sensitization effect was modulated by PCP sensitization. One-way ANOVA followed by post hoc LSD tests revealed that rats previously sensitized with SAL+NIC had significantly higher motor activity in comparison to the $\mathrm{SAL}+\mathrm{SAL} / \mathrm{SAL}$ group, $p<0.012$. Iptakalim at both doses (10 and $20 \mathrm{mg} / \mathrm{kg})$ did not decrease the nicotine-induced hyperlocomotion or the expression of nicotine sensitization. Rats previously sensitized with $\mathrm{PCP}+\mathrm{NIC}$ also showed significantly higher motor activity than the SAL+SAL/SAL rats when tested under saline and iptakalim $20 \mathrm{mg} / \mathrm{kg}$ conditions, $p<0.006$, but not under iptakalim $10 \mathrm{mg} / \mathrm{kg}$ condition, $p=0.207$, suggesting that iptakalim $10 \mathrm{mg} / \mathrm{kg}$ reduced the nicotine-induced hyperlocomotion to some extent. This observation was also supported by the finding that the PCP+NIC/IPT 10 group exhibited significantly lower motor activity than the $\mathrm{PCP}+\mathrm{NIC} / \mathrm{SAL}$ group, $p=0.045$. In addition, rats previously sensitized with $\mathrm{PCP}+\mathrm{SAL}$ showed significantly higher motor activity than the SAL+SAL/SAL rats, $p=0.009$ when they were tested under saline condition, indicating a possible cross-sensitization from PCP to nicotine. This significant effect disappeared under iptakalim 10 and $20 \mathrm{mg} / \mathrm{kg}$ conditions, $p>0.236$. The overall pattern of the results indicates that iptakalim was more effective in decreasing nicotine-induced increase in motor activity in PCP sensitized rats than saline rats.

During the second 30 minutes of testing, there was still a main effect of nicotine, $F(1,52)=15.821, p<0.001$, a main effect of $P C P, F(1,52)=6.957, p=0.011$, and a significant $P C P \times$ nicotine interaction, $F(1,52)=8.568$, $p=0.005$. One-way ANOVA followed by post hoc LSD tests revealed that only rats previously sensitized with SAL+NIC showed significantly higher motor activity in comparison to the SAL+SAL/SAL group, $p<0.011$. Comparing each of the three subgroups sensitized to
SAL+NIC to the corresponding subgroups sensitized to $\mathrm{PCP}+\mathrm{NIC}$ revealed a significant between-group difference under the saline and iptakalim 10 test conditions, $p<0.021$, suggesting that co-administration of PCP with nicotine attenuated the nicotine sensitization effect during this test period.

In the last 30 minutes, three-way ANOVA revealed a main effect of nicotine, $F(1,52)=11.258, p=0.001$, a significant $P C P \times$ nicotine interaction, $F(1,52)=6.645$, $p=0.013$, and iptakalim $\times$ nicotine interaction, $F(2$, $52)=5.634, p=0.006$. Group comparisons revealed that in comparison to the SAL+SAL/SAL group, rats previously sensitized with SAL+NIC showed significantly higher motor activity when tested under saline and iptakalim 20 $\mathrm{mg} / \mathrm{kg}$ conditions, $p<0.041$. Interestingly, iptakalim 20 $\mathrm{mg} / \mathrm{kg}$ potentiated nicotine-induced hyperlocomotion in rats previously sensitized to $\mathrm{PCP}+\mathrm{NIC}$. Rats in the $\mathrm{PCP}+\mathrm{NIC} / \mathrm{IPT} 20$ group showed significantly higher motor activity than the rats in the SAL $+\mathrm{SAL} / \mathrm{SAL}$ group, $p=0.030$. They also had significantly higher activity than those sensitized to $\mathrm{PCP}+\mathrm{NIC}$ but tested under saline or iptakalim $10 \mathrm{mg} / \mathrm{kg}, p<0.018$.

\section{Iptakalim Test on Nicotine-induced Hyperlocomotion in a Novel Environment}

In this 30 minutes test, once again, iptakalim showed a selective inhibitory effect on nicotine-induced hyperlocomotion in rats that were previously sensitized to $\mathrm{PCP}+\mathrm{NIC}$. It had little effect in rats previously sensitized to other combinations of drugs. Three-way ANOVA revealed a main effect of nicotine, $F(1,52)=7.613, p=0.008$, a significant $P C P \times$ nicotine interaction, $F(1,52)=8.020$, $p=0.007$. The main effect of iptakalim was also significant, $F(2,52)=5.041, p=0.010$. Post hoc LSD tests indicated that iptakalim $10 \mathrm{mg} / \mathrm{kg}$ differed significantly from saline, $p=0.004$; whereas iptakalim $20 \mathrm{mg} / \mathrm{kg}$ differed marginally from the saline, $p=0.051$. Group comparisons using one-way ANOVA followed by post hoc LSD tests revealed that in comparison to the $\mathrm{SAL}+\mathrm{SAL} / \mathrm{SAL}$ group, rats previously sensitized with SAL+NIC showed significantly higher motor activity when tested under saline and iptakalim $20 \mathrm{mg} / \mathrm{kg}$ conditions, $p<0.024$. This significant group difference was abolished by iptakalim $10 \mathrm{mg} / \mathrm{kg}, p=0.802$. Rats previously sensitized with $\mathrm{PCP}+\mathrm{NIC}$ also showed significantly higher motor activity than the SAL+SAL/SAL rats when tested under saline condition, $p=0.029$, but not under iptakalim 10 or 20 $\mathrm{mg} / \mathrm{kg}$ condition, $p>0.598$, suggesting that iptakalim decreased the nicotine-induced increase in motor activity. 

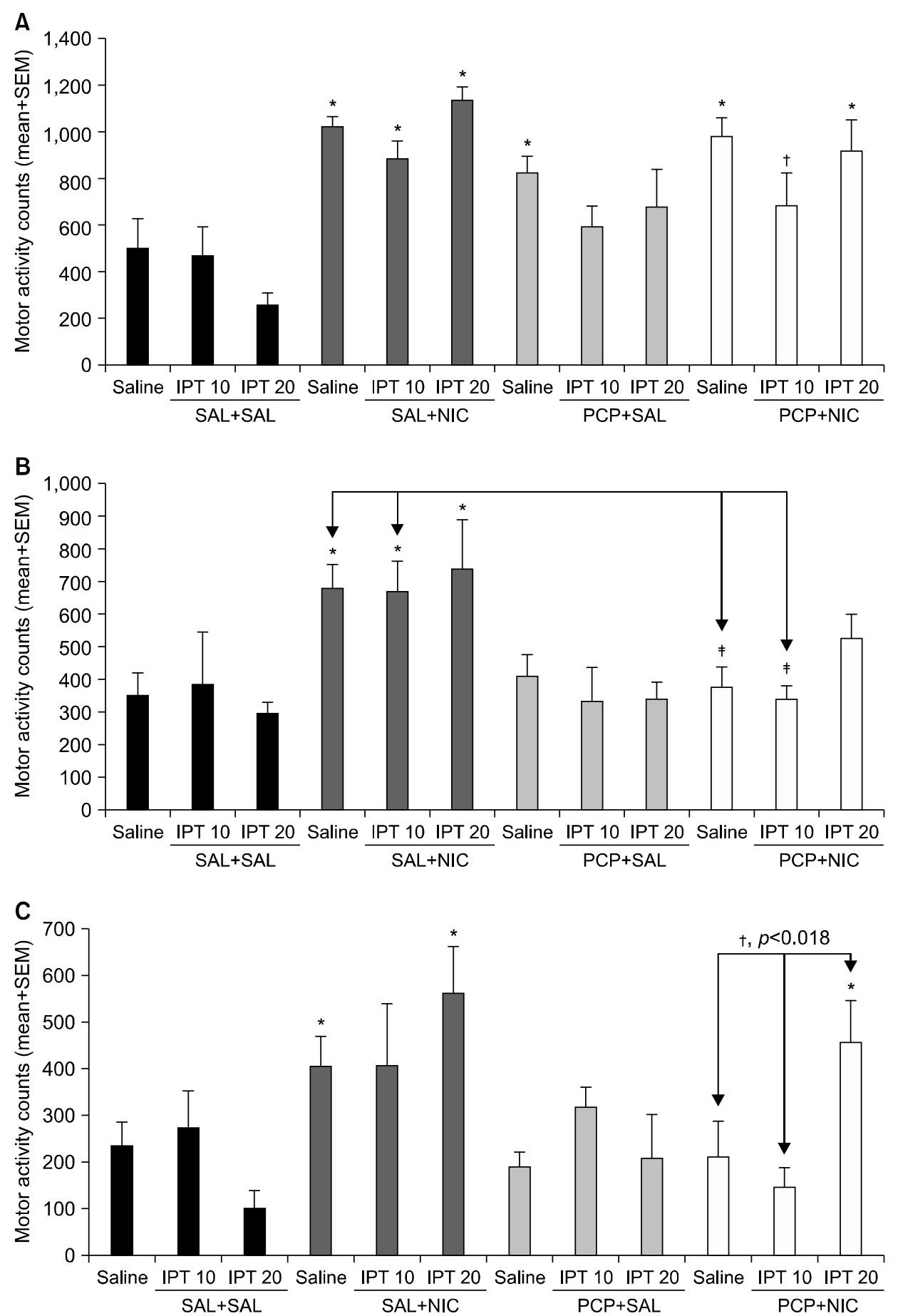

Fig. 4. Effects of iptakalim treatment (IPT; 0,10 and $20 \mathrm{mg} / \mathrm{kg}$, ip) on the expression of nicotine locomotor sensitization in the familiar environment during the nicotine challenge test in the four groups of rats $(n=16 /$ group) that were previously treated with $S A L+S A L, S A L+N I C$, $\mathrm{PCP}+\mathrm{SAL}$, and PCP+NIC. Each rat was injected with nicotine $0.2 \mathrm{mg} / \mathrm{kg}$, sc and locomotor activity was recorded for 90 minutes. Locomotor activity (mean+SEM) was separated into (A) first 30 minutes, (B) second 30 minutes, and (C) last 30 minutes. * $P<0.05$ significantly different from the SAL+SAL/SAL control group; ${ }^{\dagger} p<0.05$ significantly different between an iptakalim group and its $S A L$ control; ${ }^{\dagger} p<0.05$ significantly different between one of the SAL+NIC groups and one of the PCP+NIC groups. SAL, saline; PCP, phencyclidine; NIC, nicotine; SEM, standard error of the mean. 
The inhibitory effect of iptakalim was confirmed by the finding that the PCP+NIC/IPT 10 and PCP+NIC/IPT 20 groups showed significantly lower motor activity than the $\mathrm{PCP}+\mathrm{NIC} / \mathrm{SAL}$ group, $p<0.029$.

\section{DISCUSSION}

This present study investigated the potential therapeutic effect of iptakalim on nicotine use in schizophrenia. We created a rat model of comorbid nicotine addiction in schizophrenia using a sensitization regimen in which rats were being repeatedly injected with a combination of nicotine $(0.40 \mathrm{mg} / \mathrm{kg}, \mathrm{sc})$ and PCP $(3.0 \mathrm{mg} / \mathrm{kg}, \mathrm{sc})$ for 14 consecutive days. We confirmed the nicotine and PCP sensitization during the induction phase (i.e., 14 days of repeated drug injection) as well as in the expression phase (i.e., in the challenge tests). Because behavioral sensitization induced by a drug of abuse is often thought to reflect the addictive potential of the drug, ${ }^{28)}$ the confirmation of nicotine sensitization indicates that certain features of nicotine addiction had been successfully modeled. PCP sensitization is often regarded as a faithful model of schizophrenia. ${ }^{11,14,29,30)}$ The demonstration of persistent PPI deficits induced by PCP sensitization suggests that PCP-sensitized rats exhibited a schizophrenialike cognitive deficit, as PPI deficit is one of hallmark features associated with schizophrenia and is thought to contribute to its various symptoms (e.g., sensory flooding and cognitive fragmentation). ${ }^{31,32)}$ Overall, the 14-day sensitization regimen used in this study seems valid in capturing certain aspects of behavioral symptoms associated with nicotine use in schizophrenia. This allowed us to examine the effects of iptakalim on nicotine-induced hyperlocomotion as a way to assess its therapeutic potential against nicotine addiction in schizophrenia. We found that pretreatment of iptakalim significantly and dose-dependently reduced nicotine-induced hyperlocomotion. This effect lasted for approximately 30 minutes and was found in two distinct testing environments. The iptakalim's attenuation effect on nicotine-induced hyperlocomotion was also selective as it was more effective in rats previously sensitized to the combination of PCP and nicotine, but less effective in non-sensitized rats or those sensitized to nicotine alone or PCP alone.

In the present study, we found two interesting interactions between nicotine and PCP. Acute treatment of nicotine reduced PCP-induced hyperlocomotion and PCPnduced disruption of PPI, whereas repeated treatment of nicotine potentiated both effects of PCP (Figs. 1, 2).
Previous work on mice also found that acute nicotine ameliorates the PCP-induced deficit of PPI, ${ }^{33,34)}$ and decreases PCP-induced hyperactivity $^{35)}$ in some strains of mice. This acute reversal effect of nicotine may be related to the well-documented acute motor suppressive effect of nicotine ${ }^{20,36,37)}$ and is likely mediated by the reversal of the blocking effect of PCP on nAchRs. ${ }^{35)}$ We are not aware of any work that has reported the potentiated effect of repeated nicotine treatment on repeated PCP-induced hyperlocomotion and PPI impairment. Result on the motor activity indicates a clear additive effect between these two drugs as they share a common psychomotor stimulation effect. The PPI result is not so easy to understand because nicotine alone did not significantly disrupt PPI (Fig. 2), and chronic nicotine treatment actually increases baseline PPI in Sprague-Dawley rats. ${ }^{38,39)}$ Mechanistically, both nicotine and PCP have a common action in increasing extracellular dopamine release in the nucleus accumbens . ${ }^{40-43)}$ Nicotine does so by stimulating nicotinic cholinergic receptors in the ventral tegmental area and the nucleus accumbens, ${ }^{44)}$ and PCP does so by primarily blocking glutamatergic N-methyl-D-aspartate (NMDA) receptors . ${ }^{45-47)}$ Thus repeated nicotine treatment may potentiate PCP-induced hyperlocomotion by enhancing PCP-induced increase in dopamine release in the nucleus accumbens. ${ }^{48)}$ Alternatively, because both VTA and the nucleus accumbens receive direct glutamatergic input from the prefrontal cortex and action of PCP in this area has been implicated in the regulation of its psychomotor effect, ${ }^{49)}$ it is also possible that repeated nicotine potentiates PCP-induced hyperlocomotion by enhancing PCP's activity in the prefrontal cortex, e.g., potentiating PCP-induced increase in glutamate release in the prefrontal cortex. ${ }^{13,50,51)}$ In addition to increasing dopamine release in the prefrontal cortex, ${ }^{49,52)}$ both nicotine and PCP also cause an excessive glutamate release in the prefrontal cortex ${ }^{50,51)}$ and PCP's noncompetitive antagonist actions on NMDA receptors and non-NMDA receptors are thought to mediate its PPI-disruptive effect. ${ }^{14)}$ Therefore, it is possible that the potentiated disruption of PCP-induced PPI deficit by repeated nicotine treatment is due to the additive effect of both drugs' actions on the glutamate system in the prefrontal cortex.

The main finding of the present study was that iptakalim dose-dependently reduced nicotine-induced hyperlocomotion. This finding is to some extent expected as evidence from electrophysiological and microdialysis studies shows that iptakalim selectively inhibits $\alpha 4 \beta 2$ nAChRs, ${ }^{53)}$ and antagonizes nicotine-induced increase in 


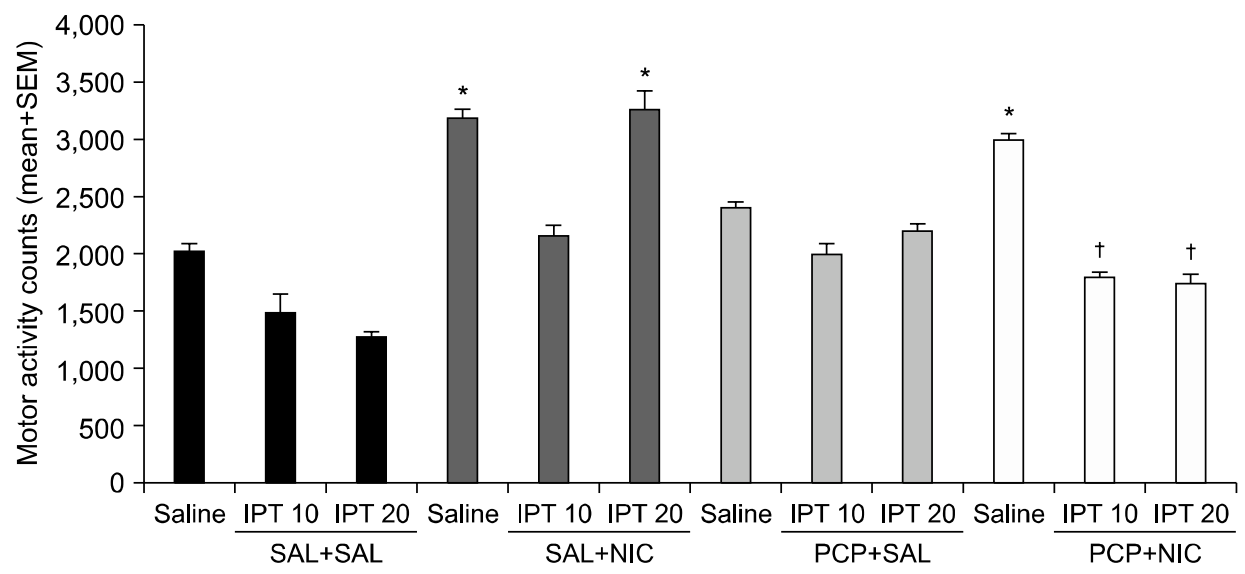

Fig. 5. Effects of iptakalim treatment (IPT; 0,10 and $20 \mathrm{mg} / \mathrm{kg}$, ip) on the expression of nicotine locomotor sensitization in the novel environment during the nicotine challenge test in the four groups of rats $(n=16 /$ group) that were previously treated with $S A L+S A L$, $S A L+N I C$, $\mathrm{PCP}+\mathrm{SAL}$, and PCP+NIC. Each rat was injected with nicotine $0.2 \mathrm{mg} / \mathrm{kg}$, sc and locomotor activity (mean+SEM) was recorded for $30 \mathrm{minutes}$ in a new testing apparatus different from the one used in the inducton of NIC/PCP sensitization. ${ }^{*} p<0.05$ significantly different from the $\mathrm{SAL}+\mathrm{SAL} / \mathrm{SAL}$ control group; ${ }^{\dagger} p<0.05$ significantly different between an iptakalim group and its saline control. SAL, saline; $\mathrm{PCP}$, phencyclidine; NIC, nicotine.

dopamine release in the accumbens. ${ }^{9)}$ Our finding provides additional behavioral support for iptakalim's inhibitory action on nicotinic receptors. The novel finding is its preferential effect in $\mathrm{PCP}+\mathrm{NIC}$ sensitized rats: iptakalim was more efficacious in decreasing nicotine-induced increase in motor activity in PCP sensitized rats than in non-sensitized rats or rats sensitized to nicotine alone or PCP alone. During the first 30 minutes of testing in a familiar environment, iptakalim $10 \mathrm{mg} / \mathrm{kg}$ only significantly decreased nicotine-induced hyperlocomotion in rats previously sensitized to the combination of PCP and nicotine, but not in rats previously sensitized to saline, nicotine alone or PCP alone. The same pattern was found during the second test in a novel environment. Because nicotine challenge induced as much higher motor activity in rats sensitized to nicotine alone as in those sensitized to both nicotine and PCP (Figs. 4A, 5), the preferential effect of iptakalim could not be attributed to different levels of motor response to nicotine challenge.

Because iptakalim is also effective in reducing PCP-induced LMA, ${ }^{10)}$ and attenuates excessive dopamine and glutamate release in the nucleus accumbens induced by nicotine and cocaine, ${ }^{9,54,55)}$ this preferential action of iptakalim may be mediated by its preferential action against excessive dopamine and glutamate release induced by nicotine. As discussed in our previous study, ${ }^{10)}$ iptakalim might do so by opening the $\mathrm{K}_{\text {ATP }}$ channels (the putative target sites of iptakalim). Opening of $\mathrm{K}_{\text {ATP }}$ channels is known to result in hyperpolarization of the cell membrane and limitation of $\mathrm{Ca}^{2+}$ influx, blocking subsequent neurotoxic biochemical cascades ${ }^{56)}$ and reducing neurotransmitter release. ${ }^{7)}$ Alternatively, recent receptor binding tests indicate that iptakalim may have an inhibitory action against sigma 2 and 1 receptors (with 97\% and 81\% inhibition rates as measured in the radioligand binding assays, thanks to National Institute of Mental Health Psychoactive Drug Screening Program). Because both nicotine and PCP effects have been suggested to be mediated at least partially by sigma receptors, ${ }^{57-59)}$ iptakalim may thus decrease nicotine-induced hyperlcomotion by inhibiting sigma receptors, as well as antagonizing nicotinic receptors. In addition, iptakalim may also antagonize $\beta_{1}$ adrenergic receptor ( $86 \%$ inhibition rate), $\mu$ opioid receptor (75\% inhibition rate) as well as $\Delta$ opioid receptor (67\% inhibition rate). This multi-receptor binding profile of iptakalim may explain the unexpected result that iptakalim $20 \mathrm{mg} / \mathrm{kg}$ seems less effective than iptakalim $10 \mathrm{mg} / \mathrm{kg}$ in decreasing nicotine-induced hyperlocmotion. We speculate that with the increase of dose, iptakalim may act on more types of receptors, raising the possibility of diluting its main action against nicotine. Obviously, this possibility can be addressed in future studies using selective agonists again these potential targets of iptakalim.

We should point out several limitations with the present study. First, we only used the LMA model to identify the anti-nicotine property of iptakalim. Other models such as nicotine-induced conditioned place preference or intravenous nicotine self-administration should also be used. Second, we did not examine the molecular mechanisms responsible for iptakalim effects in this model. As mentioned above, iptakalim may target multiple receptors or molecules to achieve its inhibitory effect on nicotine. 
Third, we have not compared iptakalim with other nicotinic receptor antagonists such as mecamylamine in this study, thus how it may differ from other drugs as pharmacotherapy for nicotine addiction remain to be determined. Finally, our chosen animal model could not capture many aspects of schizophrenia; therefore the clinical implication of our findings awaits further investigation. Nevertheless, the present study provides important preliminary evidence that iptakalim may possess a dual action against nicotine and PCP. This property is extremely important given that managing substance abuse including nicotine addiction is suggested to be a key target of schizophrenia treatment. ${ }^{60)}$ If iptakalim's therapeutic potentials are confirmed, it would contribute to broader understanding of the neuropathophysiology of co-morbidity of nicotine addiction in schizophrenia.

\section{Acknowledgments}

This study was funded by a research grant from the Nebraska Health and Human Services (2010-2011) awarded to Professor Ming Li (Principal Investigator) and Professor Rick Bevins (Co-Investigator). The funding sources have no involvement in the study design; in the collection, analysis and interpretation of data; in the writing of the report; and in the decision to submit the paper for publication. We thank Professor Rick Bevins for providing guidance on the choice of nicotine dose.

\section{REFERENCES}

1. Dalack GW, Healy DJ, Meador-Woodruff JH. Nicotine dependence in schizophrenia: clinical phenomena and laboratory findings. Am J Psychiatry 1998;155:1490-1501.

2. Lyon ER. A review of the effects of nicotine on schizophrenia and antipsychotic medications. Psychiatr Serv 1999; 50:1346-1350.

3. Gallagher SM, Penn PE, Schindler E, Layne W. A comparison of smoking cessation treatments for persons with schizophrenia and other serious mental illnesses. J Psychoactive Drugs 2007;39:487-497.

4. Lichtermann D, Ekelund J, Pukkala E, Tanskanen A, Lönnqvist J. Incidence of cancer among persons with schizophrenia and their relatives. Arch Gen Psychiatry 2001;58:573578.

5. McEvoy J, Freudenreich O, McGee M, VanderZwaag C, Levin E, Rose J. Clozapine decreases smoking in patients with chronic schizophrenia. Biol Psychiatry 1995;37:550552 .

6. Wang $\mathrm{H}$, Long $\mathrm{CL}$, Zhang YL. A new ATP-sensitive potassium channel opener reduces blood pressure and reverses cardiovascular remodeling in experimental hypertension. J Pharmacol Exp Ther 2005;312:1326-1333.

7. Wang S, Hu LF, Zhang Y, Sun T, Sun YH, Liu SY, et al. Effects of systemic administration of iptakalim on extracellular neurotransmitter levels in the striatum of unilateral 6-hydroxydopamine-lesioned rats. Neuropsychopharmacology 2006;31:933-940.
8. Hu J, Lindenberger $\mathrm{K}, \mathrm{Hu} \mathrm{G}$, Wang $\mathrm{H}$, Lukas RJ, Wu J. Iptakalim as a human nicotinic acetylcholine receptor antagonist. J Pharmacol Exp Ther 2006;316:914-925.

9. Liu Q, Li Z, Ding JH, Liu SY, Wu J, Hu G. Iptakalim inhibits nicotine-induced enhancement of extracellular dopamine and glutamate levels in the nucleus accumbens of rats. Brain Res 2006;1085:138-143.

10. Sun T, Zhao C, Hu G, Li M. Iptakalim: a potential antipsychotic drug with novel mechanisms? Eur J Pharmacol 2010; 634:68-76.

11. Sun T, Hu G, Li M. Repeated antipsychotic treatment progressively potentiates inhibition on phencyclidine-induced hyperlocomotion, but attenuates inhibition on amphetamineinduced hyperlocomotion: relevance to animal models of antipsychotic drugs. Eur J Pharmacol 2009;602:334-342.

12. Li M, Fletcher PJ, Kapur S. Time course of the antipsychotic effect and the underlying behavioral mechanisms. Neuropsychopharmacology 2007;32:263-272.

13. Jentsch JD, Roth RH. The neuropsychopharmacology of phencyclidine: from NMDA receptor hypofunction to the dopamine hypothesis of schizophrenia. Neuropsychopharmacology 1999;20:201-225.

14. Geyer MA, Krebs-Thomson K, Braff DL, Swerdlow NR. Pharmacological studies of prepulse inhibition models of sensorimotor gating deficits in schizophrenia: a decade in review. Psychopharmacology (Berl) 2001;156:117-154.

15. Jentsch JD, Tran A, Le D, Youngren KD, Roth RH. Subchronic phencyclidine administration reduces mesoprefrontal dopamine utilization and impairs prefrontal corticaldependent cognition in the rat. Neuropsychopharmacology 1997; 17:92-99.

16. Lee PR, Brady DL, Shapiro RA, Dorsa DM, Koenig JI. Social interaction deficits caused by chronic phencyclidine administration are reversed by oxytocin. Neuropsychopharmacology 2005;30:1883-1894.

17. Turgeon SM, Lin T, Subramanian M. Subchronic phencyclidine exposure potentiates the behavioral and c-Fos response to stressful stimuli in rats. Pharmacol Biochem Behav 2007;88:73-81.

18. Perkins KA. Nicotine self-administration. Nicotine Tob Res 1999;1 Suppl 2:S133-S137.

19. Clarke PB, Kumar R. The effects of nicotine on locomotor activity in non-tolerant and tolerant rats. Br J Pharmacol 1983;78:329-337.

20. Li M, Mead A, Bevins RA. Individual differences in responses to nicotine: tracking changes from adolescence to adulthood. Acta Pharmacol Sin 2009;30:868-878.

21. Shoaib M, Stolerman IP, Kumar RC. Nicotine-induced place preferences following prior nicotine exposure in rats. Psychopharmacology (Berl) 1994;113:445-452.

22. Shram MJ, Funk D, Li Z, Lê AD. Periadolescent and adult rats respond differently in tests measuring the rewarding and aversive effects of nicotine. Psychopharmacology (Berl) 2006;186:201-208.

23. Levin ED, Kaplan S, Boardman A. Acute nicotine interactions with nicotinic and muscarinic antagonists: working and reference memory effects in the 16-arm radial maze. Behav Pharmacol 1997;8:236-242.

24. Culm KE, Hammer RP Jr. Recovery of sensorimotor gating without $G$ protein adaptation after repeated D2-like dopamine receptor agonist treatment in rats. $J$ Pharmacol Exp Ther 2004;308:487-494.

25. Chen J, Wang Z, Li M. Multiple 'hits' during postnatal and early adulthood periods disrupt the normal development of sensorimotor gating ability in rats. $J$ Psychopharmacol 
2011;25:379-392.

26. Li M, He W, Mead A. Olanzapine and risperidone disrupt conditioned avoidance responding in phencyclidine-pretreated or amphetamine-pretreated rats by selectively weakening motivational salience of conditioned stimulus. Behav Pharmacol 2009;20:84-98.

27. Tenn CC, Kapur S, Fletcher PJ. Sensitization to amphetamine, but not phencyclidine, disrupts prepulse inhibition and latent inhibition. Psychopharmacology (Berl) 2005;180:366376.

28. Wolf ME. The role of excitatory amino acids in behavioral sensitization to psychomotor stimulants. Prog Neurobiol 1998;54:679-720.

29. Phillips M, Wang C, Johnson KM. Pharmacological characterization of locomotor sensitization induced by chronic phencyclidine administration. J Pharmacol Exp Ther 2001; 296:905-913.

30. Xu X, Domino EF. Phencyclidine-induced behavioral sensitization. Pharmacol Biochem Behav 1994;47:603-608.

31. Swerdlow NR, Braff DL, Geyer MA. Animal models of deficient sensorimotor gating: what we know, what we think we know, and what we hope to know soon. Behav Pharmacol 2000;11:185-204.

32. Braff DL, Geyer MA. Sensorimotor gating and schizophrenia. Human and animal model studies. Arch Gen Psychiatry 1990;47:181-188.

33. Andreasen JT, Andersen KK, Nielsen EØ, Mathiasen L, Mirza NR. Nicotine and clozapine selectively reverse a $P C P$-induced deficit of PPI in BALB/CByJ but not NMRI mice: comparison with risperidone. Behav Brain Res 2006; 167:118-127.

34. Spielewoy C, Markou A. Strain-specificity in nicotine attenuation of phencyclidine-induced disruption of prepulse inhibition in mice: relevance to smoking in schizophrenia patients. Behav Genet 2004;34:343-354.

35. Chaturvedi AK. Effects of mecamylamine, nicotine, atropine and physostigmine on the phencyclidine-induced behavioral toxicity. Pharmacol Biochem Behav 1984;20:559-566.

36. Bevins RA, Palmatier MI. Nicotine-conditioned locomotor sensitization in rats: assessment of the US-preexposure effect. Behav Brain Res 2003;143:65-74.

37. Laviolette SR, van der Kooy D. The motivational valence of nicotine in the rat ventral tegmental area is switched from rewarding to aversive following blockade of the alpha7subunit-containing nicotinic acetylcholine receptor. Psychopharmacology (Berl) 2003;166:306-313.

38. Acri JB, Brown KJ, Saah MI, Grunberg NE. Strain and age differences in acoustic startle responses and effects of nicotine in rats. Pharmacol Biochem Behav 1995;50:191198.

39. Faraday MM, O'Donoghue VA, Grunberg NE. Effects of nicotine and stress on startle amplitude and sensory gating depend on rat strain and sex. Pharmacol Biochem Behav 1999;62:273-284.

40. Clarke PB. Mesolimbic dopamine activation--the key to nicotine reinforcement? Ciba Found Symp 1990;152:153162.

41. Clarke PB, Fu DS, Jakubovic A, Fibiger HC. Evidence that mesolimbic dopaminergic activation underlies the locomotor stimulant action of nicotine in rats. $J$ Pharmacol Exp Ther 1988;246:701-708.

42. Bristow LJ, Hutson PH, Thorn L, Tricklebank MD. The glycine/NMDA receptor antagonist, $R-(+)-H A-966$, blocks activation of the mesolimbic dopaminergic system induced by phencyclidine and dizocilpine $(M K-801)$ in rodents. $\mathrm{Br} J$
Pharmacol 1993;108:1156-1163.

43. Steinpreis RE, Salamone JD. The role of nucleus accumbens dopamine in the neurochemical and behavioral effects of phencyclidine: a microdialysis and behavioral study. Brain Res 1993;612:263-270.

44. Nisell M, Nomikos GG, Svensson TH. Infusion of nicotine in the ventral tegmental area or the nucleus accumbens of the rat differentially affects accumbal dopamine release. Pharmacol Toxicol 1994;75:348-352.

45. Hernandez L, Auerbach S, Hoebel BG. Phencyclidine (PCP) injected in the nucleus accumbens increases extracellular dopamine and serotonin as measured by microdialysis. Life Sci 1988;42:1713-1723.

46. Jones SM, Snell LD, Johnson KM. Phencyclidine selectively inhibits $\mathrm{N}$-methyl-D-aspartate-induced hippocampal [3H]norepinephrine release. J Pharmacol Exp Ther 1987;240:492497.

47. Javitt DC, Zukin SR. Recent advances in the phencyclidine model of schizophrenia. Am J Psychiatry 1991;148:13011308.

48. Kleijn J, Folgering JH, van der Hart MC, Rollema H, Cremers TI, Westerink BH. Direct effect of nicotine on mesolimbic dopamine release in rat nucleus accumbens shell. Neurosci Lett 2011;493:55-58.

49. Takahata R, Moghaddam B. Activation of glutamate neurotransmission in the prefrontal cortex sustains the motoric and dopaminergic effects of phencyclidine. Neuropsychopharmacology 2003;28:1117-1124.

50. Amitai N, Kuczenski R, Behrens MM, Markou A. Repeated phencyclidine administration alters glutamate release and decreases GABA markers in the prefrontal cortex of rats. Neuropharmacology 2012;62:1422-1431.

51. Gioanni Y, Rougeot C, Clarke PB, Lepousé C, Thierry AM, Vidal C. Nicotinic receptors in the rat prefrontal cortex: increase in glutamate release and facilitation of mediodorsal thalamo-cortical transmission. Eur J Neurosci 1999;11:18-30.

52. Reid MS, Fox L, Ho LB, Berger SP. Nicotine stimulation of extracellular glutamate levels in the nucleus accumbens: neuropharmacological characterization. Synapse 2000;35: 129-136.

53. Hu J, DeChon J, Yan KC, Liu Q, Hu G, Wu J. Iptakalim inhibits nicotinic acetylcholine receptor-mediated currents in dopamine neurons acutely dissociated from rat substantia nigra pars compacta. Neurosci Lett 2006;403:57-62.

54. Yang YJ, Wang QM, Hu LF, Sun XL, Ding JH, Hu G. Iptakalim alleviated the increase of extracellular dopamine and glutamate induced by 1-methyl-4-phenylpyridinium ion in rat striatum. Neurosci Lett 2006;404:187-190.

55. Liu Y, He HR, Ding JH, Gu B, Wang H, Hu G. Iptkalim inhibits cocaine challenge-induced enhancement of dopamine levels in nucleus accumbens and striatum of rats by up-regulating Kir6.1 and Kir6.2 mRNA expression. Acta Pharmacol Sin 2003;24:527-533.

56. Miki T, Seino S. Roles of KATP channels as metabolic sensors in acute metabolic changes. $J$ Mol Cell Cardiol 2005;38:917-925.

57. Takahashi S, Horikomi K, Kato T. MS-377, a novel selective sigma(1) receptor ligand, reverses phencyclidine-induced release of dopamine and serotonin in rat brain. Eur $J$ Pharmacol 2001;427:211-219.

58. Hashimoto K, Fujita Y, Iyo M. Phencyclidine-induced cognitive deficits in mice are improved by subsequent subchronic administration of fluvoxamine: role of sigma-1 receptors. Neuropsychopharmacology 2007;32:514-521.

59. Horan B, Gardner EL, Dewey SL, Brodie JD, Ashby CR Jr. 
The selective sigma(1) receptor agonist, 1-(3,4-dimethoxyphenethyl)-4-(phenylpropyl)piperazine (SA4503), blocks the acquisition of the conditioned place preference response to (-)-nicotine in rats. Eur J Pharmacol 2001;426:R1-R2.
60. Nasrallah HA, Keshavan MS, Benes FM, Braff DL, Green AI, Gur RE, et al. Proceedings and data from The Schizophrenia Summit: a critical appraisal to improve the management of schizophrenia. J Clin Psychiatry 2009;70(Suppl 1):4-46. 